\title{
Early Supper and Late Breakfast to Reduce Diabesity
}

\author{
Akbar Nikkhah \\ Faculty of Agricultural Sciences, University of Zanjan, National Elite Foundation, Iran
}

*Corresponding Author: Akbar Nikkhah, Faculty of Agricultural Sciences, University of Zanjan, National Elite Foundation, Iran

\begin{abstract}
Timing of meal consumption has received increasing research and public interests in dealing with obesity and diabetes. Obesity especially in abdominal region and diabetes are rising problems in modern human life. Practical prevention strategies are required to reduce morbidity and costs. This article describes relatively early supper and late breakfast as a practical strategy to reduce and prevent obesity and diabetes. The strategy is feasible since it has minimal challenges in practicing. The strategy should allow the body to better synchronize its internal rhythms with external rhythms.
\end{abstract}

Keywords: Breakfast, Diabetes, Eating, Obesity, Supper, Timing.

\section{PHILOSOPHY AND Discussion}

There is no countable body of literature on taking late breakfast and early supper for reducing the risk of diabetes. The objective of this article was to introduce a feasible practical strategy to reduce obesity based on taking early supper and late breakfast. Another objective was to provide a proposal for future extensive research. Timing of eating throughout the circadian period in relation to obesity and diabetes management has received increasing research and public interests [1]. Obesity and diabetes are rising human concerns as they predispose the body to a variety of health problems worldwide [25]. The terms 'early' and 'late' are relative and should not be interpreted overly much, such that supper and breakfast are still taken in their appropriate times of the day and not interfere with lunch. In other words, it is optimal to maintain a higher meal frequency while taking early supper and late breakfast. The higher meal frequency would help minimize risks from insulin resistance and diabesity.

Early supper helps to avoid overloading the body with nutrients late overnight when glucose tolerance decreases and insulin resistance increases [6,7]. Early supper also helps to have a more relaxing and healthy sleep overnight. It reduces pressures on gastrointestinal tract when the body and mind need rest. Relatively late breakfast elongates the fasting duration and helps to mobilize peripheral and visceral fats in a controlled fashion. An early exercise before breakfast should facilitate fat mobilization and energy expenditure. Such a pre-breakfast exercise would also create a natural appetite for a healthy and timely breakfast. Since the most of the time interval between supper and breakfast is spent sleeping, it has little suboptimal effects on normal routines of daily lifestyle and all age groups should be able to easily adopt the program. With the elongated fasting time, this strategy can help to prevent diabesity providing the amounts eaten are properly restricted and managed.

Care must be exercised to not overeat at both supper and breakfast to allow the real effects to be shown over time. Increasing meal frequency will be greatly helpful. This, in addition, involves fruit and food meals optimization and is related to optimal timing of meals [8,9]. A simple rule of thumb is to stop eating before feeling full at each meal [10]. This will prevent overeating and will allow the consequences to be realized within a short period of time. The result will be reduced diabesity for obese individual and prevented diabesity for normal individuals. The goal is to live on optimal rhythms of life and be adequately close to natural rhythms of life [3,5]. Successful practice of the strategy requires victorious public education [11]. Education may start from different schools as early as possible and continue for higher age groups [12]. This article serves as a proposal for designing future research and shedding more light on the theories discussed. 


\section{CONCLUSION}

This article described a feasible strategy to reduce and prevent diabetes and obesity based on taking early supper and late breakfast. The strategy is feasible since it has minimal challenges in practicing. The strategy should allow the body to better synchronize its internal rhythms with external rhythms.

\section{ACKNOWLEDGMENTS}

Thanks to the Ministry of Science Research and Technology, and National Elite Foundation for supporting the author's global programs of optimizing science edification in the third millennium.

\section{REFERENCES}

[1] Mattson MP, Allison DB, Fontana L, Harvie M, Longo VD, Malaisse WJ, Mosleyj M, Notterpek L, Ravussin E, Scheer FAJL, Seyfried TN, Varady KA, and Panda S. 2014. Meal frequency and timing in health and disease. PNAS. 111(47): 16647-16653.

[2] Nikkhah A. 2015. Evening Exercise: A Global Strategy to Prevent Central Adiposity and Crdiometabolic Diseases. Adv. Weigh. Manag. Obes. Cont. In Press.

[3] Nikkhah A. 2015. Nature as an Ideal Rhythm Model for Optimal Cardiovascular physiology and Health. Int J Diabetol Vasc Dis Res. 3(2e) 1-2.

[4] Nikkhah A. 2015. Secure Weight Management via Fitting Circadian Patterns of Physical Activity, Resting and Eating. Adv. Weigh. Manag. Obes. Cont. 2(4): 23.

[5] Nikkhah A. 2015. Living on Healthy Rhythms to Overcome Cancer: Birth of a Public Therapeutic Science J. Nutr. Therap. In Press.

[6] Nikkhah A. 2015. Harmonizing Eating and Exercise Circadian Rhythms for Optimal Glucose-Insulin and Vascular Physiology. Int. J. Diabetol. Vasc. Dis. Res. Int J Diabetol Vasc Dis Res. 3(3): 87-88.

[7] Nikkhah A. 2015. Lifestyle Optimization: Today's Foremost Probiotic. J. Probiotics Health. 3: e119. doi:10.4172/2329-8901.1000e119.

[8] Nikkhah A. 2016. Meal Optimization to Reduce Obesity. Adv Obes Weight Manag Control. 4(5): 00105. DOI: 10.15406/aowmc.2016.04.00105.

[9] Nikkhah A. 2016. Minimizing Obesity and Diabetes by Optimizing Meal Size, Frequency, Timing and Sequence. Diabetes Obes Int J 2016, 1(5): 00012.

[10] Nikkhah A. 2016. Ceasing Eating before Feeling Full: A Turning Point in Obesity Prevention. Diab. Obes. Int. J. Diabetes Obes Int J 2016, 1(7): 000137.

[11] Nikkhah A. 2016. Science education and powerful economy. Frontiers of Marketing Research. 01(01):13.

[12] Nikkhah A. 2016. Maternal Obesity Prevention by Early Dinner and Late Breakfast. Insights from Livestock. Multidisciplinary Advances in Veterinary Science. 1(1): 27-28.

Citation: A. Nikkhah, "Early Supper and Late Breakfast to Reduce Diabesity", International Journal of Clinical Chemistry and Laboratory Medicine (IJCCLM), vol. 4, no. 1, pp. 14-15, 2018. http://dx.doi.org/10.20 431/2455-7153.0401004

Copyright: (1) 2018 Authors. This is an open-access article distributed under the terms of the Creative Commons Attribution License, which permits unrestricted use, distribution, and reproduction in any medium, provided the original author and source are credited. 